\title{
Effect of Direct Vertebral Rotation on the Uninstrumented Lumbar Curve in Thoracic Adolescent Idiopathic Scoliosis
}

\author{
Sung-Soo Kim ${ }^{1}$, Jung-Hoon $\mathrm{Kim}^{2}$, Se-Il Suk ${ }^{3}$ \\ ${ }^{1}$ Department of Orthopedic Surgery, Inje University Haeundae Paik Hospital, Busan, Korea \\ ${ }^{2}$ Department of Orthopedic Surgery, Inje University Ilsan Paik Hospital, Goyang, Korea \\ ${ }^{3}$ Department of Orthopedic Surgery, Inje University Sanggye Paik Hospital, Seoul, Korea
}

\begin{abstract}
Study Design: Retrospective study.
Purpose: To determine the effect and direction of direct vertebral rotation (DVR) in the lowest instrumented vertebra (LIV) on the uninstrumented lumbar curve depending on the lumbar modifier used for the correction of thoracic adolescent idiopathic scoliosis.

Overview of Literature: DVR in the LIV should be implemented in a different direction to obtain better spontaneous lumbar correction depending on the preoperative lumbar spine modifier.

Methods: We retrospectively analyzed 160 patients with thoracic adolescent idiopathic scoliosis treated by pedicle screw instrumentation and rod derotation. Patients who had a distal fusion level between T11 and L1 were divided into two groups: the DVR group versus the No-DVR group. Each group was divided into subgroups depending on the lumbar modifier used: the DVR-A, $B$, and $C$ groups versus the No-DVR-A, B, and C groups. The DVR-A group was subdivided into two subgroups depending on the direction of screw rotation in the LIV: the DVR-A-0 group (opposite direction) and the DVR-A-S group (same direction).

Results: There were no significant differences in the preoperative curve characteristics between the two groups. The preoperative lumbar curve was corrected in $70 \%$ of the patients in the DVR group and in $56 \%$ in the No-DVR group. Spontaneous coronal correction of the lumbar curve was better in the DVR-A-S group than that in the No-DVR-A group. However, the DVR-A-0 group had the higher incidence of adding-on deformity. The DVR-B and C groups showed better spontaneous correction of lumbar coronal magnitude, apical vertebral translation, and rotation and the LIV tilting.

Conclusions: In lumbar modifiers B and C, screws in the LIV have to be rotated opposite to the direction of the screw rotation of the main thoracic curve; however, in modifier $A$, the screws have to be rotated in the same direction.
\end{abstract}

Keywords: Spine; Scoliosis; Adolescent; Rotation; Pedicle screws

\section{Introduction}

Idiopathic scoliosis is a three-dimensional spinal deformity [1-3]. The spine shows lateral curvature in the coronal plane, thoracic hypokyphosis in the sagittal plane, and intravertebral and intervertebral rotation in the transverse plane. The ideal surgical procedure should provide maximal correction and spinal balance with minimal fusion levels. The three-dimensional deformities of both curves should be corrected.

\footnotetext{
Received Jan 24, 2017; Accepted Feb 5, 2017

Corresponding author: Jung-Hoon Kim

Department of Orthopedic Sugery, Inje University Ilsan Paik Hospital,

170 Juhwaro, Ilsanseogu, Goyang 10380, Korea

Tel: +82-31-910-7968, Fax: +82-31-910-7967, E-mail: jhkim@paik.ac.kr
} 
We have used segmental pedicle screws with a derotation maneuver in most scoliosis surgeries for the past two decades [4-7]. Our experience created some doubt concerning the success of vertebral derotation. In the late 1990s, we started to use a new method, "direct vertebral rotation (DVR)," which was designed to foster rotational correction. In 2004, we reported that better rotational and coronal correction was achieved by segmental pedicle screw fixation with DVR than by simple rod derotation [8]. A deformity in the right thoracic curve results in the apical and periapical vertebrae being rotated clockwise in the transverse plane. To correct the intervertebral rotation, the direction of DVR should be opposite to that of the rotational deformity, i.e., counter-clockwise rotation in the transverse plane. The direction of rod derotation (clockwise rotation) should be opposite to that of DVR (counter-clockwise rotation) in the apical and periapical vertebrae of the right thoracic curve (Fig. 1). The direction of DVR in the lowest instrumented vertebra (LIV) and its effect on the uninstrumented curve are still undetermined. It has been thought that the direction of DVR in the LIV might differ depending on the lumbar modifier, as described by Lenke et al. [9].

The purpose of this study was to evaluate the direction of DVR in the LIV and its effect on the uninstrumented lumbar curve depending on the lumbar modifier.

\section{Materials and Methods}

One hundred and six patients with thoracic adolescent idiopathic scoliosis were treated with pedicle screw fixation and rod derotation. All pedicle screws used were monoaxial screws. The patients were divided into two groups: patients who received DVR and patients who did not receive DVR. A retrospective analysis of the two groups was made. Fifty-four patients with DVR (DVR group) were compared with 52 patients having similar preoperative curve patterns and magnitude but who were treated with simple rod derotation without DVR (No-DVR group). All patients underwent the operation through the posterior approach and had a distal fusion level between T11 and L1. The minimum follow-up duration was 2 years (range, 2-7.7 years). According to the Lenke et al. [9] classification of curve types, the DVR group comprised 44 patients with type 1 and 10 patients with type 2, and the No-DVR group comprised 39 patients with type 1 and 13 patients with type 2 .
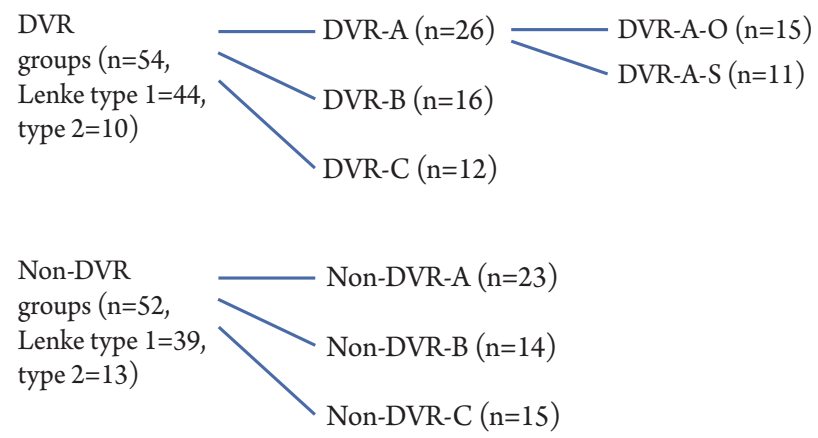

Fig. 2. Diagram of the subdivided groups. DVR, direct vertebral rotation.
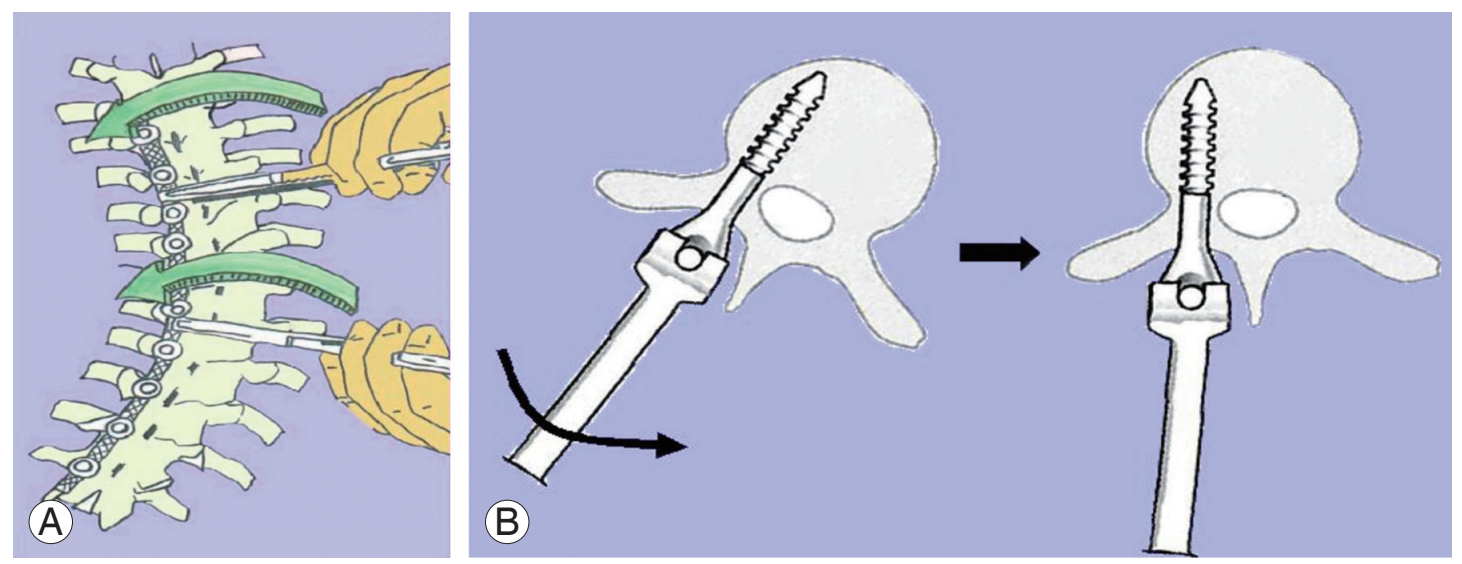

Fig. 1. (A) Diagram of rod derotation in the right thoracic curve. The rod is rotated clockwise if rod derotation is viewed from the cephalad. (B) Diagram of direct vertebral rotation in the apical and periapical vertebra of the right thoracic curve. The screw is rotated counter-clockwise to correct rotational deformity. The direction of direct vertebral rotation is opposite to that of rod derotation in the apical and periapical vertebra of the thoracic curve. 
Each group was divided into subgroups according to the lumbar modifier of Lenke (Fig. 2). A center sacral vertical line lay between the apical vertebral pedicles in lumbar modifier A, touched them in lumbar modifier B, and was completely off them in lumbar modifier C.

The DVR-A group (DVR group with lumbar modifier A) was subdivided into two groups based on the direction of screw rotation in the LIV: the DVR-A-O group in which the screw rotation direction was opposite to that in the apical and periapical vertebrae of the main thoracic curve (Fig. 3) and the DVR-A-S group in which the screw rotation direction was the same as that in the apical and periapical vertebrae of the main thoracic curve (Fig. 4). In the DVR-B group (DVR group with lumbar modifier B) and DVR-C (DVR group with lumbar modifier C) group, the screws in the LIV were rotated in the direction opposite to
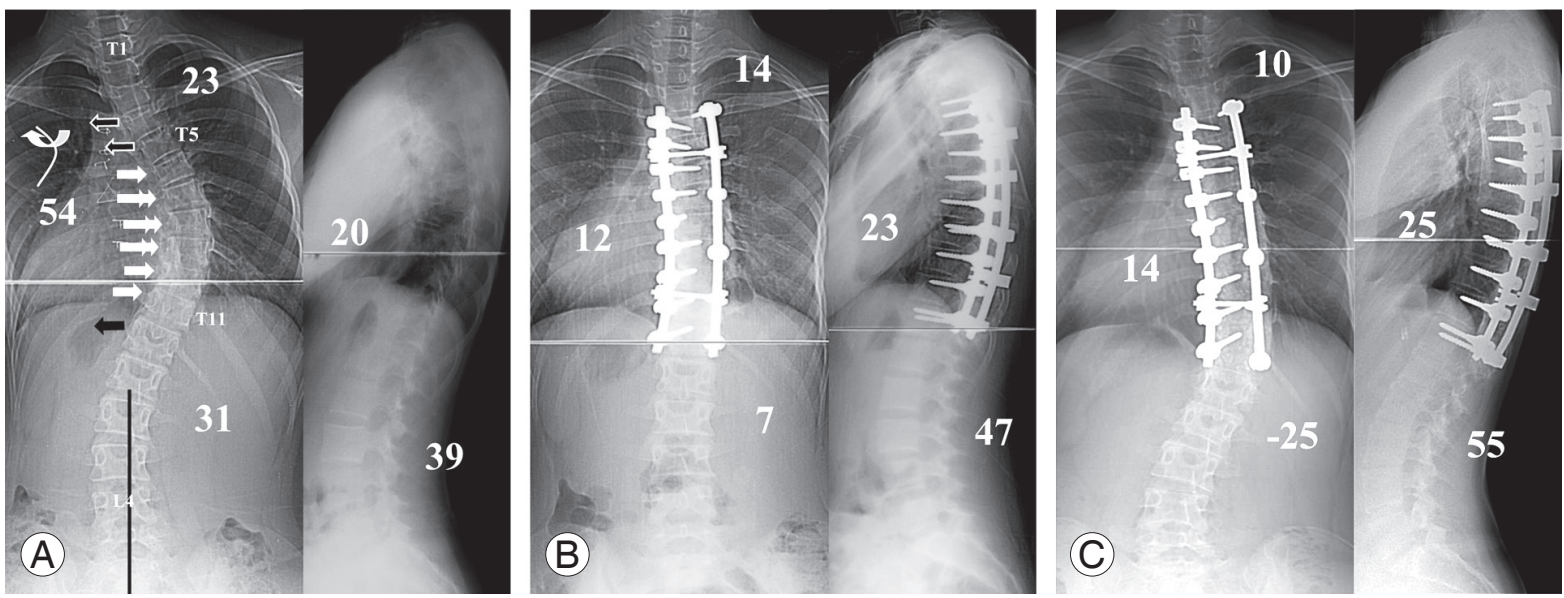

Fig. 3. A 13-year-old girl with a single thoracic curve and lumbar modifier A. (A) Preoperative anteroposterior and lateral radiographs. She was treated with pedicle screw instrumentation from T4 to T12 and rod derotation with direct vertebral rotation; round arrow=direction of rod derotation, straight arrows=directions of direct vertebral rotation. The screw in the lowest instrumented vertebra was rotated in the direction opposite to screw rotation in the apical vertebra of thoracic curve. (B) Anteroposterior and lateral radiographs taken immediately after surgery. Good correction was obtained in the thoracic and lumbar curves. (C) Anteroposterior and lateral radiographs taken 2 years and 7 months after surgery. The adding-on deformity produced during the follow-up led to the loss of lumbar curve correction.
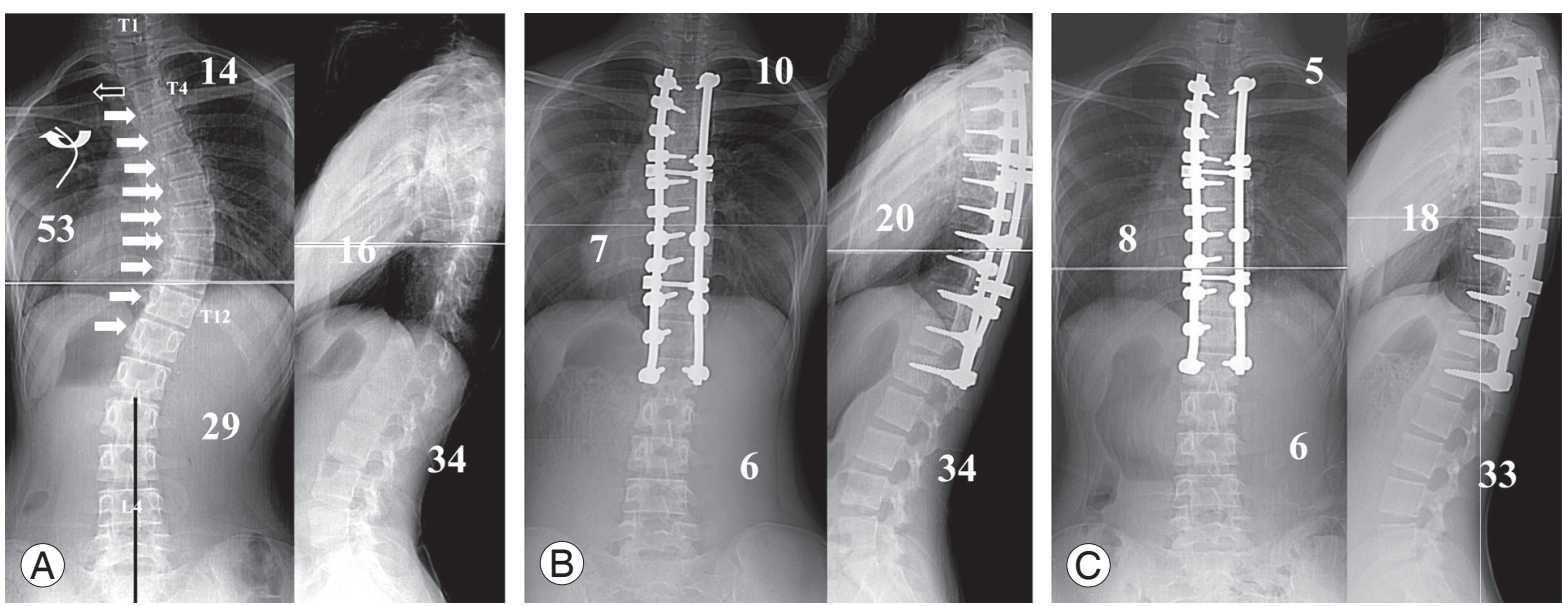

Fig. 4. A 13-year-old girl with a single thoracic curve and lumbar modifier A. (A) Preoperative anteroposterior and lateral radiographs. She was treated with pedicle screw instrumentation from T3 to $\mathrm{L} 1$ and rod derotation with direct vertebral rotation: round arrow=direction of rod derotation, straight arrows=directions of direct vertebral rotation. The screw in the lowest instrumented vertebra was rotated in the same direction as screw rotation in the apical vertebra of thoracic curve. (B) Anteroposterior and lateral radiographs taken immediately after surgery. Good correction was obtained in the thoracic and lumbar curves. (C) Anteroposterior and lateral radiographs taken 2 years after surgery. The correction was well maintained without adding-on deformity. 
screw rotation in the apical and periapical vertebrae of the main thoracic curve (Fig. 5).

In the DVR group, the average age at surgery was 15 years (range, 11-20 years), with an average follow-up duration of 3.1 years (range, $2-4.5$ years). The male-tofemale ratio was 6 to 48. In the No-DVR group, the average age was 14.9 years (range, 10.6-20 years), with an average follow-up duration of 6.4 years (range, 4.5-7.7 years). The male-to-female ratio was 4 to 48 . The curve magnitude, apical vertebral translation (AVT), apical vertebral rotation (AVR), LIV tilting, coronal and sagittal balance, and sagittal alignment were measured on a standing posteroanterior radiograph. Curve magnitude was measured using the Cobb method. The AVT of the thoracic curve was defined as the distance between a line falling from the center of C7 (C7 plumb line) and the center of the thoracic apical vertebra and that of the lumbar curve as the distance between the center sacral vertical line and the center of the lumbar apical vertebra. The AVR was checked using the Nash-Moe method and expressed by subdividing each grade into two subgrades, such as $0 \mathrm{G}$, $0.5 \mathrm{G}, 1 \mathrm{G}$, and $1.5 \mathrm{G}$. LIV tilting was defined as the angle between a parallel line to the end plate of the LIV and a horizontal line. Coronal balance was measured as the distance between the $\mathrm{C} 7$ plumb line and the center sacral vertical line. Sagittal balance was measured as the distance between the $\mathrm{C} 7$ plumb line and the posterosuperior aspect of the S1 vertebral body. Sagittal alignment was expressed by thoracic kyphosis (T5-T12) and lumbar lordosis (T12S1).

In the DVR and No-DVR groups, the preoperative and postoperative radiological parameters, such as thoracic curve, lumbar curve, LIV tilting, thoracic kyphosis, lumbar lordosis, and coronal and sagittal balance, were compared (Table 1).

The preoperative and postoperative parameters of the lumbar curve and LIV tilting were compared between the (1) DVR-A and No-DVR-A group (Table 2), (2) DVRA-O and DVR-A-S group (Table 3), (3) DVR-B and NoDVR-B group (Table 4), and (4) DVR-C and No-DVR-C group (Table 5).

\section{Surgical technique}

All surgeries were performed using the pedicle screw instrumentation system. Fusion was usually performed from one level proximal to the upper-end vertebra to one level caudal to the lower end vertebra [5,7]. Single thoracic curves (Lenke type 1) were treated with selective thoracic fusion and double thoracic curves (Lenke type 2) by double thoracic fusion. In the DVR group, the distal fusion level was T11 in four patients, T12 in 24, and L1 in 26. In the No-DVR group, the distal fusion level was T11 in five patients, T12 in 27, and L1 in 20.
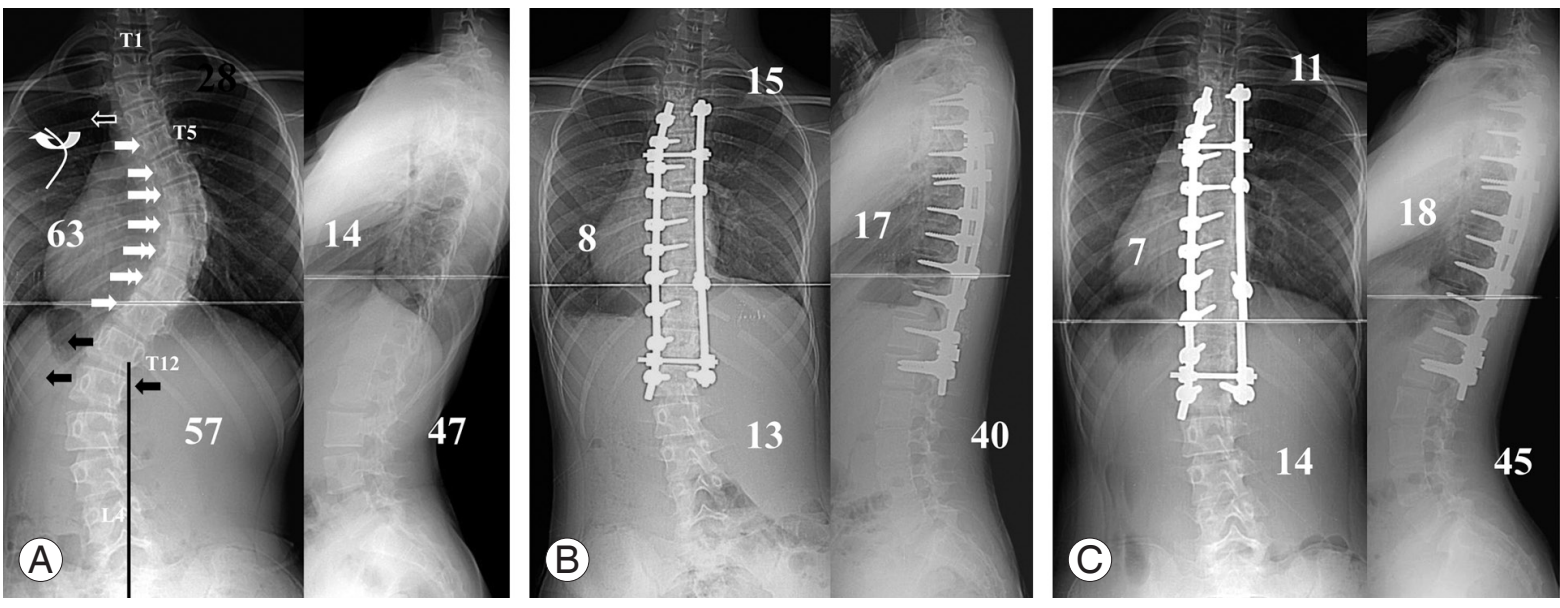

Fig. 5. A 13-year-old girl with a single thoracic curve and lumbar modifier C. (A) Preoperative anteroposterior and lateral radiographs. She was treated with pedicle screw instrumentation from T4 to $\mathrm{L} 1$ and rod derotation with direct vertebral rotation: round arrow=direction of rod derotation, straight arrows=directions of direct vertebral rotation. Three screws in the two lowermost instrumented vertebrae were rotated in the direction opposite to screw rotation in the apical vertebra of thoracic curve. (B) Anteroposterior and lateral radiographs taken immediately after surgery. Good correction was obtained in the thoracic and lumbar curves. (C) Anteroposterior and lateral radiographs taken 3 years after surgery. The correction was well maintained. 
Table 1. The results of DVR and No-DVR group

\begin{tabular}{|c|c|c|c|c|c|}
\hline \multirow{2}{*}{ Parameters } & \multicolumn{2}{|c|}{ DVR group (n=54) } & \multicolumn{2}{|c|}{ No-DVR group $(n=52)$} & \multirow{2}{*}{$p$-value } \\
\hline & Preoperative & Postoperative & Preoperative & Postoperative & \\
\hline \multicolumn{6}{|l|}{ Thoracic curve } \\
\hline Cobb angle $\left({ }^{\circ}\right)$ & $53.4 \pm 12.9$ & $14.6 \pm 8.1$ & $53.2 \pm 10.2$ & $18.2 \pm 8.2$ & 0.026 \\
\hline Coronal correction (\%) & 72.7 & & 66.0 & & 0.013 \\
\hline $\operatorname{LOC}(\%)$ & 3.3 & & 5.5 & & $>0.05$ \\
\hline $\mathrm{AVT}(\mathrm{mm})$ & $43.4 \pm 11.4$ & $11.5 \pm 7.6$ & $40.8 \pm 12.0$ & $11.3 \pm 6.8$ & $>0.05$ \\
\hline AVT correction (\%) & 73.6 & & 72.2 & & $>0.05$ \\
\hline \multicolumn{6}{|l|}{ Lumbar curve } \\
\hline Cobb angle $\left({ }^{\circ}\right)$ & $33.7 \pm 8.6$ & $10.6 \pm 6.0$ & $33.7 \pm 9.8$ & $15.4 \pm 8.9$ & 0.002 \\
\hline Coronal correction (\%) & 69.5 & & 56.4 & & 0.001 \\
\hline LOC $(\%)$ & 7.8 & & 7.3 & & $>0.05$ \\
\hline $\mathrm{AVT}(\mathrm{mm})$ & $11.6 \pm 6.5$ & $7.9 \pm 4.9$ & $13.1 \pm 8.3$ & $12.2 \pm 8.4$ & 0.002 \\
\hline AVT correction (mm) & 4.1 & & 0.4 & & 0.005 \\
\hline $\operatorname{AVR}(G)$ & $1.2 \pm 0.7$ & $0.9 \pm 0.7$ & $1.1 \pm 0.6$ & $1.0 \pm 0.7$ & $>0.05$ \\
\hline AVR correction (G) & 0.3 & & 0.1 & & $>0.05$ \\
\hline LIV tilting $\left(^{\circ}\right)$ & $20.4 \pm 7.3$ & $5.5 \pm 3.3$ & $20.9 \pm 7.2$ & $7.9 \pm 4.4$ & 0.003 \\
\hline LIV tilting correction (\%) & 74.9 & & 62.3 & & 0.002 \\
\hline Thoracic kyphosis $\left({ }^{\circ}\right)$ & $17.2 \pm 9.9$ & $26.7 \pm 8.3$ & $17.8 \pm 9.1$ & $27.2 \pm 7.5$ & $>0.05$ \\
\hline Lumbar lordosiss $\left({ }^{\circ}\right)$ & $43.6 \pm 9.6$ & $49.3 \pm 10.3$ & $45.5 \pm 9.8$ & $51.5 \pm 11.2$ & $>0.05$ \\
\hline Coronal balance (mm) & $9.8 \pm 7.5$ & $9.1 \pm 6.1$ & $10.9 \pm 8.8$ & $8.1 \pm 6.5$ & $>0.05$ \\
\hline Sagittal balance $(\mathrm{mm})$ & $30.0 \pm 21.0$ & $26.7 \pm 19.3$ & $26.7 \pm 17.0$ & $21.5 \pm 15.6$ & $>0.05$ \\
\hline
\end{tabular}

Values are presented as mean \pm standard deviation.

DVR, direct vertebral rotation; LOC, loss of curve correction during the follow-up; AVT, apical vertebral translation; AVR, apical vertebral rotation; LIV, lowest instrumented vertebra.

The technique of the surgical procedure in the DVR groups (Fig. 1) was as follows. After posterior exposure and facetectomy, pedicle screws were inserted segmentally on the correction side (thoracic concavity) and every other or third vertebra on the support side (thoracic convexity). A precontoured rod was inserted into the correction side and rotated in a clockwise direction (from the cephalad view) without any compression or distraction. Specially designed screw derotators (4-8 derotators) were connected to the heads of the pedicle screws in the apical and periapical vertebrae on the correction side. After rod derotation, the connected screw derotators were rotated to improve the rotation of the apical and periapical vertebrae, opposite to the rod derotation. In one or two uppermost instrumented vertebrae, screws were rotated to correct the rotation of the upper uninstrumented curve, usually clockwise and opposite to the apical and periapical screw rotation. Screw rotation in the LIV was dependent on the preoperative lumbar modifier. For lumbar modifier A, screw rotation in the LIV was in the opposite direction (Fig. 3) or same direction (Fig. 4) to that of thoracic DVR, as stated above. In lumbar modifiers B and C (Fig. 5), the screw in the LIV was rotated clockwise in the direction opposite to that of the thoracic DVR to correct vertebral rotation (counter-clockwise) of the lumbar curve. In modifier $\mathrm{C}$, the screws in two LIVs were rotated with greater torque. After locking the concave rod in the corrected position, the other rod contoured to the corrected curve was inserted into the convex side and locked in place. Intraoperative portable radiographs were taken to evaluate the instrumented thoracic curve and uninstrumented lumbar curve. If the coronal and rotational corrections of the lumbar curve were inadequate, both screws in the LIV were unlocked and rotated further to obtain better 
Table 2. The lumbar curve and LIV tilting of DVR-A and No-DVR-A group

\begin{tabular}{|c|c|c|c|}
\hline Parameters & DVR-A group (n=26) & No-DVR-A group (n=23) & $p$-value \\
\hline \multicolumn{4}{|l|}{ Lumbar curve } \\
\hline Preoperative Cobb $\left({ }^{\circ}\right)$ & $29.7 \pm 9.1$ & $27.5 \pm 6.5$ & $>0.05$ \\
\hline Postoperative Cobb $\left({ }^{\circ}\right)$ & $8.2 \pm 4.9$ & $9.4 \pm 5.1$ & $>0.05$ \\
\hline Correction (\%) & 73.2 & 65.9 & $>0.05$ \\
\hline Loss of curve correction $(\%)$ & 9.2 & 4.3 & 0.043 \\
\hline Preoperative AVT (mm) & $7.0 \pm 2.9$ & $6.8 \pm 5.1$ & $>0.05$ \\
\hline Postoperative AVT (mm) & $5.5 \pm 3.0$ & $5.6 \pm 4.6$ & $>0.05$ \\
\hline AVT correction & 1.8 & 0.2 & $>0.05$ \\
\hline Preoperative AVR (G) & $0.7 \pm 0.6$ & $0.8 \pm 0.4$ & $>0.05$ \\
\hline Postoperative AVR (G) & $0.5 \pm 0.5$ & $0.6 \pm 0.7$ & $>0.05$ \\
\hline AVR correction & 0.2 & 0.2 & $>0.05$ \\
\hline \multicolumn{4}{|l|}{ LIV tilting } \\
\hline Preoperative LIV tilting $\left(^{\circ}\right)$ & $20.7 \pm 8.7$ & $19.7 \pm 5.6$ & $>0.05$ \\
\hline Postoperative LIV tilting $\left({ }^{\circ}\right)$ & $6.2 \pm 3.0$ & $5.7 \pm 2.8$ & $>0.05$ \\
\hline LIV tilting correction (\%) & 71.9 & 71.0 & $>0.05$ \\
\hline
\end{tabular}

Values are presented as mean \pm standard deviation.

LIV, lowest instrumented vertebra; DVR, direct vertebral rotation; AVT, apical vertebral translation; AVR, apical vertebral rotation.

Table 3. The lumbar curve and LIV tilting of DVR-A-O and DVR-A-S group

\begin{tabular}{|c|c|c|c|}
\hline Parameters & DVR-A-O group ( $n=15)$ & DVR-A-S group $(n=11)$ & $p$-value \\
\hline \multicolumn{4}{|l|}{ Lumbar curve } \\
\hline Preoperative Cobb $\left({ }^{\circ}\right)$ & $31.4 \pm 9.7$ & $27.6 \pm 8.3$ & $>0.05$ \\
\hline Postoperative Cobb $\left(^{\circ}\right)$ & $9.6 \pm 4.7$ & $6.3 \pm 4.7$ & 0.048 \\
\hline Correction (\%) & 69.8 & 77.4 & $>0.05$ \\
\hline Loss of curve correction (\%) & 15.3 & 2.1 & 0.043 \\
\hline Preoperative AVT (mm) & $7.3 \pm 3.0$ & $6.6 \pm 2.8$ & $>0.05$ \\
\hline Postoperative AVT (mm) & $6.2 \pm 3.6$ & $4.5 \pm 0.4$ & $>0.05$ \\
\hline AVT correction & 1.5 & 2.1 & $>0.05$ \\
\hline Preoperative AVR (G) & $0.9 \pm 0.6$ & $0.5 \pm 0.5$ & $>0.05$ \\
\hline Postoperative AVR (G) & $0.6 \pm 0.6$ & $0.4 \pm 0.4$ & $>0.05$ \\
\hline AVR correction & 0.3 & 0.0 & $>0.05$ \\
\hline \multicolumn{4}{|l|}{ LIV tilting } \\
\hline Preoperative LIV tilting $\left(^{\circ}\right)$ & $21.8 \pm 8.8$ & $19.6 \pm 8.9$ & $>0.05$ \\
\hline Postoperative LIV tilting $\left({ }^{\circ}\right)$ & $6.7 \pm 3.6$ & $5.6 \pm 1.9$ & $>0.05$ \\
\hline LIV tilting correction (\%) & 76.7 & 67.5 & $>0.05$ \\
\hline
\end{tabular}

Values are presented as mean \pm standard deviation.

LIV, lowest instrumented vertebra; DVR, direct vertebral rotation; AVT, apical vertebral translation; AVR, apical vertebral rotation.

correction. In lumbar modifier $\mathrm{C}$, the screws in the two lowermost instrumented vertebrae were used for DVR. After finishing DVR, the two rods were usually joined by two transverse connectors. Then, posterior fusion was performed with bone graft. 
Table 4. The lumbar curve and LIV tilting of DVR-B and No-DVR-B group

\begin{tabular}{|c|c|c|c|}
\hline Parameters & DVR-B group (n=16) & No-DVR-B group (n=14) & $p$-value \\
\hline \multicolumn{4}{|l|}{ Lumbar curve } \\
\hline Preoperative Cobb $\left({ }^{\circ}\right)$ & $35.6 \pm 4.9$ & $36.1 \pm 9.9$ & $>0.05$ \\
\hline Postoperative Cobb $\left({ }^{\circ}\right)$ & $11.4 \pm 6.8$ & $17.4 \pm 9.6$ & 0.044 \\
\hline Correction (\%) & 69.1 & 52.7 & 0.026 \\
\hline Loss of curve correction $(\%)$ & 6.6 & 8.7 & $>0.05$ \\
\hline Preoperative AVT (mm) & $14.7 \pm 3.5$ & $15.4 \pm 4.5$ & $>0.05$ \\
\hline Postoperative AVT (mm) & $10.1 \pm 5.4$ & $16.0 \pm 3.5$ & 0.004 \\
\hline AVT correction & 4.6 & 0.6 Aggravation & 0.013 \\
\hline Preoperative AVR (G) & $1.6 \pm 0.4$ & $1.3 \pm 0.5$ & $>0.05$ \\
\hline Postoperative AVR (G) & $1.3 \pm 0.6$ & $1.1 \pm 0.5$ & $>0.05$ \\
\hline AVR correction & 0.3 & 0.1 & $>0.05$ \\
\hline \multicolumn{4}{|l|}{ LIV tilting } \\
\hline Preoperative LIV tilting $\left(^{\circ}\right)$ & $20.4 \pm 5.4$ & $24.1 \pm 6.9$ & $>0.05$ \\
\hline Postoperative LIV tilting $\left({ }^{\circ}\right)$ & $3.7 \pm 2.2$ & $8.0 \pm 5.3$ & 0.013 \\
\hline LIV tilting correction (\%) & 81.4 & 65.5 & 0.033 \\
\hline
\end{tabular}

Values are presented as mean \pm standard deviation.

LIV, lowest instrumented vertebra; DVR, direct vertebral rotation; AVT, apical vertebral translation; AVR, apical vertebral rotation.

Table 5. The lumbar curve and LIV tilting of DVR-C and No-DVR-C group

\begin{tabular}{|c|c|c|c|}
\hline Parameters & DVR-C group (n=12) & No-DVR-C group $(n=15)$ & $p$-value \\
\hline \multicolumn{4}{|l|}{ Lumbar curve } \\
\hline Preoperative Cobb ( $\left.{ }^{\circ}\right)$ & $39.6 \pm 7.8$ & $41.5 \pm 7.8$ & $>0.05$ \\
\hline Postoperative Cobb $\left({ }^{\circ}\right)$ & $15.0 \pm 4.3$ & $22.7 \pm 6.9$ & 0.004 \\
\hline Correction (\%) & 61.7 & 45.3 & 0.008 \\
\hline Loss of curve correction (\%) & 6.3 & 10.4 & $>0.05$ \\
\hline Preoperative AVT (mm) & $19.4 \pm 6.7$ & $22.8 \pm 5.0$ & $>0.05$ \\
\hline Postoperative AVT (mm) & $10.8 \pm 5.4$ & $19.1 \pm 8.2$ & 0.020 \\
\hline AVT correction & 8.7 & 1.8 & 0.042 \\
\hline Preoperative AVR (G) & $1.8 \pm 0.4$ & $1.4 \pm 0.6$ & $>0.05$ \\
\hline Postoperative AVR (G) & $1.2 \pm 0.7$ & $1.6 \pm 0.5$ & $>0.05$ \\
\hline AVR correction & 0.5 & 0.1 Aggravation & 0.016 \\
\hline \multicolumn{4}{|l|}{ LIV tilting } \\
\hline Preoperative LIV tilting $\left(^{\circ}\right)$ & $19.7 \pm 7.1$ & $22.3 \pm 6.9$ & $>0.05$ \\
\hline Postoperative LIV tilting $\left({ }^{\circ}\right)$ & $6.4 \pm 4.4$ & $11.0 \pm 4.3$ & 0.019 \\
\hline LIV tilting correction (\%) & 70.9 & 47.5 & 0.015 \\
\hline
\end{tabular}

Values are presented as mean \pm standard deviation.

LIV, lowest instrumented vertebra; DVR, direct vertebral rotation; AVT, apical vertebral translation; AVR, apical vertebral rotation.

\section{Statistical analysis}

All statistical analyses were processed on a personal computer running commercially available software (MedCalc, Belgium). Parametric (Student's t-test) and nonparametric analyses (Mann-Whitney U-test and Kruskal- 
Wallis test) were used, and statistical significance was set at $p<0.05$.

\section{Results}

\section{DVR versus No-DVR groups}

The preoperative and postoperative radiological parameters of these groups are shown in Table 1. There were no statistical differences in preoperative thoracic Cobb angle and AVT, lumbar Cobb angle, AVT and AVR, LIV tilting, coronal and sagittal balance, thoracic kyphosis, and lumbar lordosis between the DVR and No-DVR groups (all $p>0.05)$. There were statistically significant differences in thoracic and lumbar curve correction, lumbar AVT correction, and the average correction of LIV tilting between the two groups. There were no significant differences in postoperative coronal and sagittal balance, thoracic kyphosis, and lumbar lordosis between the two groups.

\section{Subgroups with lumbar modifier A (DVR-A-O and DVR-A-S versus No-DVR-A)}

The preoperative and postoperative radiological parameters of these groups are shown in Tables 2 and 3. There were statistically significant differences in the loss of lumbar curve correction for the follow-up period between the DVR-A and No-DVR-A groups (Table 2). Analysis of the DVR-A group was performed by dividing it into two subgroups depending on the direction of screw rotation in the LIV. Comparison of the DVR-A-O and DVR-A-S groups showed that there was no significant difference in lumbar curve correction postoperatively, but there was a statistically significant difference in the loss of lumbar curve correction during the follow-up period (Table 3 ).

\section{Subgroups with lumbar modifier B (DVR-B versus No-DVR-B)}

The preoperative and postoperative radiological parameters of these groups are shown in Table 4. There were statistically significant differences in the lumbar curve and AVT corrections when comparing the lumbar characteristics between the two groups. The overall AVR correction was better in the DVR-B group; however, the difference was not significant. There was a statistically significant difference in the average correction of LIV tilting.

\section{Subgroups with lumbar modifier C (DVR-C versus No-DVR-C)}

The preoperative and postoperative radiological parameters of these groups are shown in Table 5. There were statistically significant differences in lumbar coronal, AVT, and AVR corrections when comparing the lumbar characteristics between the two groups. There was a statistically significant difference in the average correction of LIV tilting.

\section{Complications}

Progression of the curve caudal to the instrumented fusion (adding-on) was detected in 11 patients at the latest follow-up: seven in DVR-A-O (7/15, 45\%), one in DVRA-S $(1 / 11,9 \%)$, one in DVR-B $(1 / 16,6 \%)$, one in DVR-C $(1 / 12,8 \%)$, and one in the No-DVR-A group $(1 / 23,4 \%)$. There was a high risk of postoperative adding-on in the DVR-A-O group compared with that in the other groups. In the DVR-A-O group, three patients and seven patients had adding-on at the immediate postoperative period and at the latest follow-up, respectively. The risk of postoperative adding-on was correlated with the loss of spontaneous lumbar curve correction (Fig. 3); however, this loss was related neither to coronal imbalance nor to an increased incidence of back pain during follow-up. Additionally, there were neither neurological nor vascular complications related to the use of pedicle screws.

\section{Discussion}

Idiopathic scoliosis is a complex three-dimensional spinal deformity in the coronal, sagittal, and transverse planes [1-3]. The strong postero-medialization of rod derotation is known to provide three-dimensional correction and has been generally used for treating idiopathic scoliosis $[4,5,10,11]$. However, there is a controversy regarding its rotational correction.

Pollock and Pollock [12] reported that $30^{\circ}$ of the rotational correction could be achieved by using hooks. Lenke et al. [13] showed $11^{\circ}$ of rotational improvement, but Bridwell [14] reported that only a limited amount of rotational correction could be achieved. Gardner-Morse and Stokes [1] reported that $8^{\circ}$ of vertebral rotation was aggravated after rod derotation using an FEM model. Several reports have stated that rod derotation had a minor influence on rotational correction $[8,12,15,16]$. These 
reported studies demonstrated that the posterior hook instrumentation system could not generate sufficient torque for improving the vertebral rotation because the axis of the hook was posterior to that of vertebral rotation. Compared with the hook, the pedicle screw can control the anterior spinal column because it effectively uses not only the pedicle but also the vertebral body as an anchor for fixation. Our experience over time with pedicle screw fixation for idiopathic scoliosis has led the authors to conclude that the rotational force needed to correct intervertebral rotation could be transmitted through the pedicle screw from the posterior pedicle to the anterior vertebral body. This corrective force in the transverse plane can be successfully generated by maneuvering the long lever-arm screw derotator tightly connected to the pedicle screw. Following up on this idea, the authors introduced DVR in idiopathic scoliosis surgery to obtain better three-dimensional correction, particularly rotational correction, in the late 1990s. In previous papers, the authors reported that a thoracic AVR of $42.5 \%$ could be corrected with DVR, whereas that of $2.4 \%$ with simple rod derotation and DVR gave a true three-dimensional deformity correction in idiopathic scoliosis surgery and provided better coronal and rotational correction [8,17-19]. In the present study, coronal correction of the fused thoracic curve was better in the DVR group. The thoracic AVT correction was similar between the DVR and No-DVR groups. There has been no evidence about the direction of screw derotation in the LIV.

Screws in the LIV have an important role in regulating the compensatory lumbar curve. At the beginning of DVR, screws in the LIV were rotated to improve the rotation of the unfused lumbar curve depending on the preoperative standing radiograph. For example, screws in the LIV were rotated clockwise if the compensatory lumbar curve was rotated counter-clockwise. However, there were several cases involving adding-on deformities during the follow-up, even if the patients showed excellent deformity corrections immediately after surgery. The authors had carefully reviewed the preoperative, intraoperative, and postoperative radiographs and found that adding-on deformities correlated with the preoperative compensatory lumbar curve.

When the center sacral vertical line falls laterally to the medial aspect of the lumbar apical pedicle (lumbar modifier B or C) with significant opposite rotation relative to the main thoracic curve, the compensatory lumbar curve can be spontaneously corrected by screw rotation in the LIV, which improves the lumbar rotation. For example, screws in the LIV were rotated clockwise because the lumbar curve with modifier B or C had a counter-clockwise rotation in the right thoracic curve. This maneuver provides better deformity correction in both the coronal and transverse planes than does simple rod derotation. In our series, the DVR-B and C groups showed better coronal correction and AVT in the unfused lumbar curve than the No-DVR-B and C groups. Moreover, rotational correction in the lumbar correction was better in the case of DVR, although AVR was assessed by using the Nash-Moe method. There was a minor difference in DVR between lumbar modifiers $\mathrm{B}$ and $\mathrm{C}$. The lumbar curve with modifier $\mathrm{B}$ could be corrected by one (concave side) or two screw rotations in the LIV only. However, the lumbar modifier $\mathrm{C}$ curve needed more rotational force to spontaneously correct the lumbar curve. Thus, three screws (two in the concave and one in the convex side) in the two lowermost instrumented vertebrae were used for correcting lumbar curve with more forceful torque. Hence, the authors differentiated lumbar modifier B from C in this study.

Further, when the center sacral vertical line falls between the lumbar apical pedicles (lumbar modifier A), DVR in the LIV should be different from that in lumbar modifier B or C. Because this was not noticed at the beginning of DVR, the screw of the LIV in lumbar modifier A was rotated in the same direction as that of lumbar modifier B or C. Given that adding-on deformity increased during the follow-up, the authors focused on the direction of DVR in the LIV and performed it by using the other way to reduce that problem.

The lumbar curve in lumbar modifier A is flexible, and its apical vertebra has a minor rotation. In this case, rod derotation and DVR in the apical and periapical vertebrae could straighten the lumbar curve. Subsequently, if DVR in the LIV was performed in the direction opposite (DVR-A-O) to that in the apical and periapical vertebrae (thoracic DVR), the lumbar curve could be overcorrected; this caused the distal extension of the fused thoracic curve during the follow-up. In our study, compared with the DVR-A-S group, the DVR-A-O group had a high risk of postoperative adding-on even if it showed better rotational correction of the lumbar curve. The adding-on was found in seven patients $(7 / 15,45 \%)$ in the DVR-A-O group at the latest follow-up and was related to the loss of spontaneous lumbar correction during the follow-up. 
The authors suggest that DVR in the LIV with the same direction (DVR-A-S) as that in the periapical vertebrae should prevent postoperative adding-on by balancing the unfused lumbar rotation with the fused thoracic rotation. In our series, the DVR-A-S group had adding-on in one patient $(1 / 11,9 \%)$, a considerably lower incidence rate than that in the DVR-A-O group, with satisfactory correction. Adding-on was detected in two patients in the DVR-B and C groups (one in DVR-B and one in DVR-C), whereas there was no patient with adding-on in the NoDVR-B and C groups. This result may be because of the powerful correctional force of DVR, which indicates that surgeons should perform DVR in the LIV carefully while taking intraoperative radiographs.

There were several limitations in this study. Distal adding-on could be influenced by not only the direction of DVR in the LIV but also by the LIV level and LIV tilt. The number of patients in the DVR-A group was too small to allow analysis of other factors, but this analysis would be possible in a future study that collects more data from a larger study population. We neither checked the torque of screw rotation nor expressed its exact amount in lumbar modifier B or C. This issue also could be a good project in the near future. However, it is obvious that lumbar modifier $\mathrm{C}$ required more forceful screw rotation in the LIV than did lumbar modifier B to improve spontaneous correction, as described above.

\section{Conclusions}

DVR in the LIV should be implemented in different directions, depending on the preoperative lumbar spine modifier used, to obtain better spontaneous lumbar correction. In lumbar modifiers B and C, screws in the LIV must be rotated opposite to the direction of screw rotation of the main thoracic curve. However, in modifier A, screws in the LIV must be rotated in the same direction as screw rotation of the main thoracic curve.

\section{Conflict of Interest}

No potential conflict of interest relevant to this article was reported.

\section{References}

1. Gardner-Morse M, Stokes IA. Three-dimensional simulations of the scoliosis derotation maneuver with Cotrel-Dubousset instrumentation. J Biomech 1994; 27:177-81.

2. Matsumoto T, Kitahara H, Minami S, et al. Flexibility in the scoliotic spine: three-dimensional analysis. J Spinal Disord 1997;10:125-31.

3. Perdriolle R, Vidal J. Morphology of scoliosis: threedimensional evolution. Orthopedics 1987;10:909-15.

4. Suk SI, Lee CK, Chung SS. Comparison of Zielke ventral derotation system and Cotrel-Dubousset instrumentation in the treatment of idiopathic lumbar and thoracolumbar scoliosis. Spine (Phila Pa 1976) 1994;19:419-29.

5. Suk SI, Lee CK, Kim WJ, Chung YJ, Park YB. Segmental pedicle screw fixation in the treatment of thoracic idiopathic scoliosis. Spine (Phila Pa 1976) 1995; 20:1399-405.

6. Suk SI, Kim WJ, Lee SM, Kim JH, Chung ER. Thoracic pedicle screw fixation in spinal deformities: are they really safe? Spine (Phila Pa 1976) 2001;26:204957.

7. Suk SI, Lee SM, Chung ER, Kim JH, Kim SS. Selective thoracic fusion with segmental pedicle screw fixation in the treatment of thoracic idiopathic scoliosis: more than 5-year follow-up. Spine (Phila Pa 1976) 2005;30:1602-9.

8. Lee SM, Suk SI, Chung ER. Direct vertebral rotation: a new technique of three-dimensional deformity correction with segmental pedicle screw fixation in adolescent idiopathic scoliosis. Spine (Phila Pa 1976) 2004;29:343-9.

9. Lenke LG, Betz RR, Harms J, et al. Adolescent idiopathic scoliosis: a new classification to determine extent of spinal arthrodesis. J Bone Joint Surg Am 2001; 83:1169-81.

10. Cotrel Y, Dubousset J, Guillaumat M. New universal instrumentation in spinal surgery. Clin Orthop Relat Res 1988;227:10-23.

11. Muschik M, Schlenzka D, Robinson PN, Kupferschmidt C. Dorsal instrumentation for idiopathic adolescent thoracic scoliosis: rod rotation versus translation. Eur Spine J 1999;8:93-9.

12. Pollock FE, Pollock FE Jr. Idiopathic scoliosis: correction of lateral and rotational deformities using the Cotrel-Dubousset spinal instrumentation system. South Med J 1990;83:161-5.

13. Lenke LG, Bridwell KH, Baldus C, Blanke K, Schoe- 
necker PL. Cotrel-Dubousset instrumentation for adolescent idiopathic scoliosis. J Bone Joint Surg Am 1992;74:1056-67.

14. Bridwell KH. Surgical treatment of adolescent idiopathic scoliosis: the basics and the controversies. Spine (Phila Pa 1976) 1994;19:1095-100.

15. Krismer M, Bauer R, Sterzinger W. Scoliosis correction by Cotrel-Dubousset instrumentation: the effect of derotation and three dimensional correction. Spine (Phila Pa 1976) 1992;17:S263-9.

16. Labelle H, Dansereau J, Bellefleur C, de Guise J, Rivard $\mathrm{CH}$, Poitras B. Peroperative three-dimensional correction of idiopathic scoliosis with the CotrelDubousset procedure. Spine (Phila Pa 1976) 1995;20: 1406-9.
17. Mladenov KV, Vaeterlein C, Stuecker R. Selective posterior thoracic fusion by means of direct vertebral derotation in adolescent idiopathic scoliosis: effects on the sagittal alignment. Eur Spine J 2011;20:11147.

18. Di Silvestre M, Lolli F, Bakaloudis G, Maredi E, Vommaro F, Pastorelli F. Apical vertebral derotation in the posterior treatment of adolescent idiopathic scoliosis: myth or reality? Eur Spine J 2013;22:313-23.

19. Fu G, Kawakami N, Goto M, Tsuji T, Ohara T, Imagama S. Comparison of vertebral rotation corrected by different techniques and anchors in surgical treatment of adolescent thoracic idiopathic scoliosis. J Spinal Disord Tech 2009;22:182-9. 